Research Article

\title{
DOA Tracking of Two-Dimensional Coherent Distribution Source Based on Fast Approximated Power Iteration
}

\author{
Tao Wu $\mathbb{D}^{1},{ }^{1}$ Pengtao Zhang, ${ }^{1}$ Yiwen Li, ${ }^{2}$ Yangjun Gao, ${ }^{1}$ Chaoqi Fu, ${ }^{1}$ and Bo Feng ${ }^{1}$ \\ ${ }^{1}$ Equipment Management and UAV College, Air Force Engineering University, Xi'an 710051, Shaanxi, China \\ ${ }^{2}$ Science and Technology on Combustion, Thermal-Structure and Internal Flow Laboratory, \\ Northwestern Polytechnical University, Xi'an 710051, Shaanxi, China \\ Correspondence should be addressed to Tao Wu; taowu_nwpu@126.com
}

Received 1 August 2020; Revised 9 September 2020; Accepted 29 September 2020; Published 16 November 2020

Academic Editor: Wang Zheng

Copyright ( $\odot 2020$ Tao Wu et al. This is an open access article distributed under the Creative Commons Attribution License, which permits unrestricted use, distribution, and reproduction in any medium, provided the original work is properly cited.

\begin{abstract}
Aiming at two-dimensional (2D) coherent distributed (CD) sources, this paper has proposed a direction of arrival (DOA) tracking algorithm based on signal subspace updating under the uniform rectangular array (URA). First, based on the hypothesis of small angular spreads of distributed sources, the rotating invariant relations of the signal subspace of the receive vector of URA are derived. An ESPRIT-like method is constructed for DOA estimation using two adjacent parallel linear arrays of URA. Through the synthesis of estimation by multiple groups of parallel linear arrays within URA arrays, the DOA estimation method for 2D CD sources based on URA is obtained. Then, fast approximated power iteration (FAPI) subspace tracking algorithm is used to update the signal subspace. In this way, DOA tracking of $2 \mathrm{D}$ CD sources can be realized by DOA estimation through signal subspace updating. This algorithm has a low computational complexity and good real-time tracking performance. In addition, the algorithm can track multiple $\mathrm{CD}$ sources without knowing the angular signal distribution functions, which is robust to model errors.
\end{abstract}

\section{Introduction}

In the field of underwater acoustic and radar detection, since the position of target is constantly changing, it is necessary to obtain the target orientation information in real time. The traditional DOA tracking methods assume that the target is a point source. Considering stationary targets based on the point source model, some important achievements have been made in the field of mixed signal and sparse array and multiangle estimation recently. Based on the uniform rectangular array (URA), the two-dimensional (2D) direction of arrival (DOA) estimation of circular and noncircular mixed signals is proposed in [1]. In [2], authors have proposed a reduced dimension noncircular MUSIC algorithm for DOA and polarization estimation of circular and noncircular mixed signals in the virtual multiple input multiple output (MIMO) system. The authors of [3] have proposed two kinds of extended coprime arrays, namely, sliding extended coprime array and coprime array with displaced subarrays, which can alleviate mutual coupling and increased degree of freedom significantly. Based on a coprime array, authors of [4] have proposed an estimator where the Toeplitz matrix functions is utilized to resolve off-grid sources. The authors of [5] have realized the estimation of 2D DOA, receiving and transmitting angle simultaneously in the electromagnetic MIMO system. The authors of [6] have presented direction of departure and DOA estimation for the MIMO radar where a reduced dimension multiple signal classification algorithm requiring one-dimension search is derived. The authors of [7] have proposed an estimator for DOA and mutual coupling self-calibration in the uniform linear arrays-based bistatic MIMO radar where a two-step framework is proposed for DOA estimation, and mutual coupling coefficients are obtained via the least square method. In [8], authors have proposed an estimator for joint 2D DOD, 2D DOA, and polarization angles without pairing in the bistatic MIMO system with electromagnetic vector sensors. 
There are two main types of DOA tracking methods based on the point source model. One is a mature method based on the Kalman filter $[9,10]$, which has been used to track the parameters of DOA, target distance, and so on. The other is based on signal subspace updating methods, such as projection approximation subspace tracking (PAST) and PAST based on the deflation technique (PASTd) [11-13], orthonormal projection approximation subspace tracking (OPAST) $[14,15]$, and fast approximated power iteration (FAPI) [16, 17]. In the field of underwater acoustic detection, due to the existence of multipath propagation between the receiving array and target, especially with the reduction of the distance between the target and receiving array, the spatial scattering characteristics of the target cannot be ignored, and the DOA estimation or tracking algorithm based on the point source model is no longer established. Under this context, scholars put forward the distributed source models [18].

According to the time correlation of scattering elements, distributed sources can be divided into incoherent and coherent sources. The incoherent distributed (ID) source assumes that different scattering elements of a target are incoherent, and the coherent distributed (CD) source assumes that different scattering elements of a target are coherent. According to the spatial distribution dimension, the distributed sources can be classified into one-dimensional (1D) and two-dimensional (2D) distributed sources. The 2D distributed sources assume that the target and receiving array are in a three-dimensional space, which is more general. For CD sources, the spatial distribution function describing the scattering elements of the target is a deterministic angular signal distribution function (ASDF), which is a probability density function depending on the distribution of scattering points in the space.

As the distributed source model not only includes all the parameters of the point source but also adds the angular spread parameters, the complexity of parameter estimation increases significantly. For 2D ASDF function, it can be modeled as Gaussian, uniform, or other function forms. The parameters included are the nominal azimuth, nominal elevation, azimuth spreads, and elevation spreads. Nominal azimuth and nominal elevation can be collectively called DOA reflecting direction of arrival of the target center.

Considering the targets are static, scholars have presented different algorithms to model and solve DOA estimation of distributed sources under diverse arrays [19-30]. Nevertheless, in the background of a moving target, the DOAs of distributed sources are time-varying. The research studies on DOA tracking of distributed sources are relatively fewer. Based on the covariance matrix renewed by the exponential window function, literature [31] has presented a DOA tracking method of $1 \mathrm{D} C D$ sources where the signal subspace is updated by FAPI; then, DOA is resolved by TLSESPRIT. Authors of [32] have presented a DOA tracking model based on the support vector machine (SVM) for 1D $\mathrm{CD}$ sources where vectorizations of the covariance matrix renewed by the exponential window function are regarded as the input of SVM, and DOAs are used as the output. In literature [33], an adaptive DOA tracking method for 1D ID sources is proposed where DOAs are estimated by the covariance fitting technique using the central moments of the angular power density; then, utilizing the constant acceleration model, the DOA tracking model is established in the framework of the Kalman filter.

This paper presents a DOA tracking algorithm for $2 \mathrm{D}$ CD sources based on URA. First, the rotating invariant relations of signal subspace are derived by exploring the URA structure under the premise of small angular spreads of sources. Then, the signal subspace is updated by FAPI tracking algorithm in real time. Based on the rotation invariant relations of the signal subspace, DOAs of $2 \mathrm{D} C D$ sources can be calculated. This algorithm has a low computational complexity and good real-time tracking performance. In addition, the algorithm can track multiple distributed sources without knowing the ASDF of distributed sources and shows robustness to model errors. The main contributions of this article are listed below.

(i) The rotation invariance relations within the URA with respect to nominal elevation and nominal azimuth are deduced under small angular spreads assumption

(ii) The 2D DOA estimation algorithm of CD sources is derived based on URA, which does not need to know the ASDF form of sources and need not spectral peak search and can be applied for DOA estimation for point sources under the background of the nonmoving target

(iii) Based on the FAPI framework, a DOA tracking method is proposed. The algorithm has good tracking accuracy and can track multiple 2D CD sources in real time. It is also suitable for DOA tracking of point sources and has robustness in the case of mismatch between the point source and CD source model.

The structure of the paper is as follows. Part 2 introduces the signal model and the receive vectors of arrays proposed. In part 3, the solution approach of DOA based on URA is detailed. Part 4 elaborates DOA tracking based on signal subspace iteration. Part 5 illustrates the simulation results. Part 6 draws the conclusion.

Notations: scalar variables are denoted by italic letters, and vectors and matrices are denoted by bold letters. $(\cdot)^{-1}$, $(\cdot)^{T}$, and $(\cdot)^{H}$ mean inverse, transpose, and Hermitian transpose of a matrix. $E[\cdot],(. /)$, and $(\cdot)+$ denote expectation, elementwise division, and pseudoinverse operation; diag $(\cdot)$ is the diagonal matrix; $\arg (\cdot)$ is the phase of a complex number.

\section{Array Structure and Signal Model}

The structure of the array is shown in Figure 1. The URA is on the $x o z$ plane. The distance between sensors along the $x$ axis and $z$-axis is $d$. There are $M$ sensors along the $x$-axis and $K$ sensors along the $z$-axis, and the array contains $M K$ sensors totally. It is assumed that there are $q 2 \mathrm{D}$ CD sources with wavelength $\lambda$ in the far-field space with DOAs as $\left(\theta_{i}, \varphi_{i}\right)$ 
$(i=1,2, \ldots, q)$ impinging on the array. $\theta_{i}$ is the nominal azimuth of the $i^{\text {th }}$ source, and $\varphi_{i}$ is the nominal elevation, $\theta_{i} \in[0, \pi], \varphi_{i} \in[0, \pi]$. It is assumed that the noise of each sensor is additive Gaussian white noise, which is not related to the signal. Assuming that the origin sensor position is $m=k=1$, the signal received by the $(m, k)^{\text {th }}$ sensor in URA can be expressed as

$$
y_{m k}(t)=\sum_{i=1}^{q} \iint e^{j \pi(m-1)(2 d \cos \theta \sin \phi / \lambda)} e^{j \pi(k-1)(2 d \cos \phi / \lambda)} s_{i}(\theta, \phi, t) d \theta d \phi+n_{m k}(t),
$$

where $n_{m k}(t)$ is the noise received. As for CD sources, the angular signal density function can be written as

$$
s_{i}(\theta, \phi, t)=s_{i}(t) g_{i}\left(\theta, \phi ; \mathbf{u}_{i}\right),
$$

where $s_{i}(t)$ reflects time characteristics of the $i^{\text {th }} \mathrm{CD}$ sources, $g_{i}\left(\theta, \varphi ; \mathbf{u}_{i}\right)$ is the deterministic angular signal density function (ASDF) of the $i^{\text {th }}$ source, and $\sigma_{i}^{2}=\left\|s_{i}(t)\right\|^{2}$ is the power of the source. Define signal vector $s(t)$ as follows:

$$
\mathbf{s}(t)=\left[s_{1}(t), s_{2}(t), \ldots, s_{q}(t)\right]^{T} .
$$

Assumed different CD sources are incoherent, we have

$$
\boldsymbol{\Lambda}=E\left[\mathbf{s}(t) \mathbf{s}(t)^{H}\right]=\operatorname{diag}\left(\sigma_{1}^{2}, \sigma_{2}^{2}, \ldots, \sigma_{q}^{2}\right) .
$$

The generalized manifold coefficient of the $(m, k)^{\text {th }}$ sensor for the $i^{\text {th }}$ source is defined as

$$
\eta_{m k}\left(\theta_{i}, \phi_{i}\right)=\iint e^{j 2 \pi d(m-1) \cos \theta \sin \phi / \lambda} e^{j 2 \pi d(k-1) \cos \phi / \lambda} g_{i}\left(\theta, \phi ; \mathbf{u}_{i}\right) d \theta d \phi .
$$

Reflecting the response to all CD sources, the generalized manifold vector of the $(m, k)^{\text {th }}$ sensor can be expressed as

$$
\mathbf{a}_{m k}(\theta, \phi)=\left[\eta_{m k}\left(\theta_{1}, \phi_{1}\right), \eta_{m k}\left(\theta_{2}, \phi_{2}\right), \ldots, \eta_{m k}\left(\theta_{q}, \phi_{q}\right)\right] .
$$

The signal received by the $(m, k)^{\text {th }}$ sensor can be expressed as

$$
y_{m k}(t)=\mathbf{a}_{m k}(\theta, \phi) \mathbf{s}(t)+n_{m k}(t) .
$$

Under the premise of small angular spreads of sources and the distance between sensors, $d$ is set as the half of the wavelength $\lambda$, generalized manifold coefficients of the $(m, k)^{\text {th }}$, the $(m-1, k)^{\mathrm{th}}$, and the $(m, k-1)^{\mathrm{th}}$, and the $(m-1, k-1)^{\mathrm{th}}$ sensors have the rotating relations as follows (Appendix):

$$
\begin{aligned}
& \eta_{m k}\left(\theta_{i}, \phi_{i}\right) \approx e^{j \pi \cos \theta_{i} \sin \phi_{i}} \eta_{(m-1) k}\left(\theta_{i}, \phi_{i}\right), \\
& \eta_{m k}\left(\theta_{i}, \phi_{i}\right) \approx e^{j \pi \cos \phi_{i}} \eta_{m(k-1)}\left(\theta_{i}, \phi_{i}\right), \\
& \eta_{m k}\left(\theta_{i}, \phi_{i}\right) \approx e^{j \pi \cos \theta_{i} \sin \phi_{i}} e^{j \pi \cos \phi_{i}} \eta_{(m-1)(k-1)}\left(\theta_{i}, \phi_{i}\right) .
\end{aligned}
$$

\section{DOA Estimation under URA}

In order to track the dynamic target in real time, DOA estimation for the 2D CD source based on URA should be derived based on the signal subspace, which is a basis of
DOA tracking. In this part, according to the rotating relations (8-10), an ESPRIT-like DOA estimation method for the 2D CD signal source is constructed between two adjacent parallel linear arrays of URA. Through the synthesis solutions of multiple groups of parallel linear arrays within URA arrays, the 2D DOA estimation method based on URA is obtained.

$M$ sensors on the $x$-axis are defined as subarray $X_{1}$, and the received signals of subarray $X_{1}$ can be expressed as

$$
\mathbf{x}_{1}(t)=\mathbf{A}_{1} \mathbf{s}(t)+\mathbf{n}_{x 1}(t),
$$

where $A_{1}$ is the generalized manifold matrix of subarray $X_{1}$, which has the following expression:

$$
\mathbf{A}_{1}=\left[\begin{array}{c}
\mathbf{a}_{11}(\theta, \phi) \\
\mathbf{a}_{21}(\theta, \phi) \\
\ldots \\
\mathbf{a}_{M 1}(\theta, \phi)
\end{array}\right]=\left[\begin{array}{cccc}
\eta_{11}\left(\theta_{1}, \phi_{1}\right) & \eta_{11}\left(\theta_{2}, \phi_{2}\right) & \cdots & \eta_{11}\left(\theta_{q}, \phi_{q}\right) \\
\eta_{21}\left(\theta_{1}, \phi_{1}\right) & \eta_{21}\left(\theta_{2}, \phi_{2}\right) & \cdots & \eta_{21}\left(\theta_{2}, \phi_{2}\right) \\
\ldots & \cdots & \cdots & \cdots \\
\eta_{M 1}\left(\theta_{1}, \phi_{1}\right) & \eta_{M 1}\left(\theta_{1}, \phi_{1}\right) & \cdots & \eta_{M 1}\left(\theta_{q}, \phi_{q}\right)
\end{array}\right] .
$$

Define subarray $X_{k}$ as the linear array parallel to the $x$ axis and with the interval of $(k-1) d$ from subarray $X_{1}$. It can be concluded that the received signal of subarray $X_{k}$ is

$$
\mathbf{x}_{k}(t)=\mathbf{A}_{k} \mathbf{s}(t)+\mathbf{n}_{k}(t),
$$

where the noise vector can be expressed as

$$
\mathbf{n}_{k}(t)=\left[n_{1, k-1}(t), n_{2, k-1}(t), \ldots, n_{M, k-1}(t)\right] .
$$

Take the first $M-1$ sensors of subarray $X_{k}$ to form subarray $\mathrm{X}_{k}^{1}$ and the last $M-1$ sensors to form subarray $\mathrm{X}_{k}^{2}$, and the generalized manifold matrix of subarray $\mathrm{X}_{k}^{1}$ can be expressed as

$$
\mathbf{A}_{k}^{1}=\left[\mathbf{I}_{(M-1) \times(M-1)} \mid 0_{(M-1) \times 1}\right] \mathbf{A}_{k} .
$$

Generalized manifold matrix of subarray $\mathrm{X}_{k}^{2}$ can be expressed as

$$
\mathbf{A}_{k}^{2}=\left[0_{(M-1) \times 1} \mid \mathbf{I}_{(M-1) \times(M-1)}\right] \mathbf{A}_{k} .
$$

According to equations (8)-(10), generalized manifold matrices have the following rotating invariant relations:

$$
\begin{aligned}
& \mathbf{A}_{k}^{2} \approx \mathbf{A}_{k}^{1} \Phi_{x}, \\
& \mathbf{A}_{k}^{1} \approx \mathbf{A}_{k-1}^{1} \boldsymbol{\Phi}_{z},
\end{aligned}
$$

where rotational operations have the following expressions: 


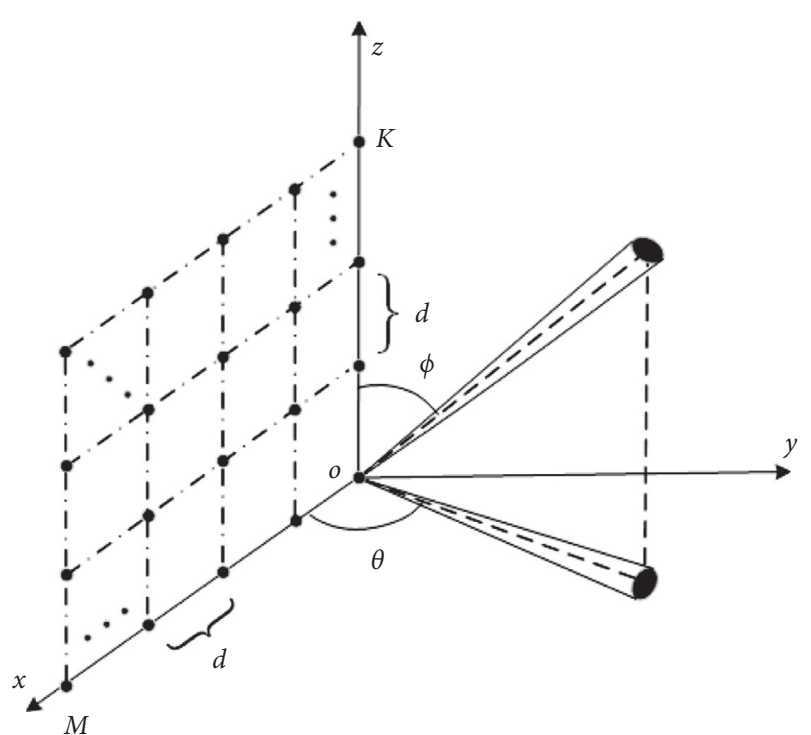

FIGURE 1: Uniform rectangular array structure.

$$
\begin{aligned}
& \boldsymbol{\Phi}_{x}=\operatorname{diag}\left(e^{j \pi \cos \theta_{1} \sin \phi_{1}}, e^{j \pi \cos \theta_{2} \sin \phi_{2}}, \ldots, e^{j \pi \cos \theta_{q} \sin \phi_{q}}\right), \\
& \boldsymbol{\Phi}_{z}=\operatorname{diag}\left(e^{j \pi \cos \phi_{1}}, e^{j \pi \cos \phi_{2}}, \ldots, e^{j \pi \cos \phi_{q}}\right) .
\end{aligned}
$$

Receive vectors of subarrays $X_{k}^{1}$ and $X_{k}^{2}$ can be written as

$$
\begin{aligned}
& \mathbf{x}_{k}^{1}(t) \approx \mathbf{A}_{k}^{1} \mathbf{s}(t)+\mathbf{n}_{k}^{1}(t), \\
& \mathbf{x}_{k}^{2}(t) \approx \mathbf{A}_{k}^{2} \mathbf{s}(t)+\mathbf{n}_{k}^{2}(t) .
\end{aligned}
$$

Receive vectors of URA can be written as

$$
\mathbf{X}(t)=\left[\begin{array}{c}
\mathbf{x}_{1}(t) \\
\mathbf{x}_{2}(t) \\
\cdots \\
\mathbf{x}_{K}(t)
\end{array}\right]=\mathbf{A s}(t)+\mathbf{n}_{X}(t)
$$

where $\mathbf{A}$ is the generalized manifold matrix of URA, which have the following expression:

$$
\mathbf{A}=\left[\begin{array}{c}
\mathbf{A}_{1} \\
\mathbf{A}_{2} \\
\cdots \\
\mathbf{A}_{K}
\end{array}\right]
$$

Receive noise vector of URA can be written as

$$
\mathbf{n}_{X}(t)=\left[\mathbf{n}_{1}(t)^{T}, \mathbf{n}_{2}(t)^{T}, \ldots, \mathbf{n}_{K}(t)^{T}\right]^{T}
$$

where

$$
\begin{aligned}
& E\left[\mathbf{n}_{X}(t) \mathbf{n}_{X}^{H}\left(t^{\prime}\right)\right]=\sigma_{n}^{2} \mathbf{I}_{M K} \delta\left(t-t^{\prime}\right), \\
& E\left[\mathbf{n}_{X}(t) \mathbf{n}_{X}^{T}\left(t^{\prime}\right)\right]=0, \quad \forall t, t^{\prime}
\end{aligned}
$$

The covariance matrix of $\mathbf{X}(t)$ can be written as

$$
\mathbf{R}_{Z}=E\left[\mathbf{X}(t) \mathbf{X}(t)^{H}\right]=\mathbf{A} \mathbf{\Lambda} \mathbf{A}^{H}+\sigma_{n}^{2} \mathbf{I}_{M K} \cdot
$$

The signal subspace $\mathrm{W}$ composed of eigenvectors corresponding to the largest $q$ eigenvalues can be obtained by eigendecomposition of $\mathbf{R}_{Z}$. The space of $\mathbf{W}$ is the same as that of $\mathbf{A}$. Therefore, a $q \times q$ dimensional nonsingular matrix $\mathbf{F}$ satisfies the following relation:

$$
\mathbf{W}=\mathbf{A F}=\left[\begin{array}{c}
\mathbf{A}_{1} \\
\mathbf{A}_{2} \\
\cdots \\
\mathbf{A}_{K}
\end{array}\right] \mathbf{F}=\left[\begin{array}{c}
\mathbf{B}_{1} \\
\vdots \\
\mathbf{B}_{\varsigma} \\
\vdots \\
\mathbf{B}_{K / 2}
\end{array}\right] \mathbf{F}
$$

where

$\mathbf{B}_{1}=\left[\begin{array}{c}\mathbf{A}_{1}^{1} \\ \text { the mth row of } \mathbf{A}_{1} \\ \mathbf{A}_{2}^{1} \\ \text { the 2mth row of } \mathbf{A}_{2}\end{array}\right]=\left[\begin{array}{c}\text { the mth row of } \mathbf{A}_{1} \\ \mathbf{A}_{1}^{2} \\ \mathbf{A}_{2}^{1} \\ \text { the 2mth row of } \mathbf{A}_{2}\end{array}\right]$,

$\mathbf{B}_{\varsigma}=\left[\begin{array}{c}\mathbf{A}_{2 \varsigma-1}^{1} \\ \text { the mth row of } \mathbf{A}_{2 \varsigma-1} \\ \mathbf{A}_{2 \varsigma}^{1} \\ \text { the } 2 \varsigma \text { th row of } \mathbf{A}_{2 \varsigma}\end{array}\right]=\left[\begin{array}{c}\text { the 1 } \text { th row of } \mathbf{A}_{2 \varsigma-1} \\ \mathbf{A}_{2 \varsigma-1}^{2} \\ \mathbf{A}_{2 \varsigma}^{1} \\ \text { the } 2 \varsigma \text { th row of } \mathbf{A}_{2 \varsigma}\end{array}\right]$.

Define $\mathbf{W}_{1}^{1}$ as a vector selecting the first $M-1$ rows of $\mathrm{W}$ and $\mathbf{W}_{1}^{2}$ as the $2^{\text {nd }}$ to $M$ th rows of $\mathbf{W} . \mathbf{W}_{2 \varsigma-1}^{1}$ is a vector selecting $[(\varsigma-1) 2 M+1]$ th to $[(\varsigma-1) 2 M+M-1]$ th rows of $\mathbf{W}, \mathbf{W}_{2 \varsigma-1}^{2}$ is a vector selecting $[(\varsigma-1) 2 M+2]$ th to $[(\varsigma-1) 2 M+M]$ of rows $\mathbf{W}$, and $\varsigma=1,2, \ldots, K / 2 ; K$ is an even number. According to equation (26), $\mathbf{W}_{2 \varsigma-1}^{1}, \mathbf{W}_{2 \varsigma-1}^{2}$, and $\mathbf{W}_{2 \varsigma}^{1}$ have the following relations:

$$
\begin{aligned}
\mathbf{W}_{2 \zeta-1}^{1} & =\mathbf{A}_{2 \zeta-1}^{1} \mathbf{F}, \\
\mathbf{W}_{2 \varsigma-1}^{2} & =\mathbf{A}_{2 \varsigma-1}^{2} \mathbf{F}, \\
\mathbf{W}_{2 \varsigma}^{1} & =\mathbf{A}_{2 \varsigma}^{1} \mathbf{F} .
\end{aligned}
$$

According to the rotating invariant relations described by equations (17) and (18), we have

$$
\begin{gathered}
\mathbf{W}_{2 \varsigma-1}^{2}\left(\mathbf{W}_{2 \varsigma-1}^{1}\right)^{+} \mathbf{A}_{2 \varsigma-1}^{1}=\mathbf{A}_{2 \varsigma-1}^{1} \boldsymbol{\Phi}_{X}, \\
\mathbf{W}_{2 \varsigma}^{1}\left(\mathbf{W}_{2 \varsigma-1}^{1}\right)^{+} \mathbf{A}_{2 \varsigma-1}^{1}=\mathbf{A}_{2 \varsigma-1}^{1} \boldsymbol{\Phi}_{Z} .
\end{gathered}
$$

It is known that the rotation operators $\Phi_{x}$ and $\Phi_{z}$ are the diagonal matrices. The corresponding eigenvectors of the elements in the same position of the diagonal matrices $\Phi_{x}$ and $\Phi_{z}$ are the same. Therefore, the eigenvalues $e^{j \pi \cos \phi_{i}}$ of $\mathbf{W}_{2 \varsigma}^{1}\left(\mathbf{W}_{2 \varsigma-1}^{1}\right)^{+}$and eigenvalues $e^{j \pi \cos \theta_{i} \sin \phi_{i}}$ of $\mathbf{W}_{2 \varsigma-1}^{2}\left(\mathbf{W}_{2 \varsigma-1}^{1}\right)^{+}$ have the same eigenvector.

Eigenvalue $e^{j \pi \cos \phi_{i}}$ can be obtained by eigendecomposition $\mathbf{W}_{2 \varsigma}^{1}\left(\mathbf{W}_{2 \varsigma-1}^{1}\right)^{+}$. Then, nominal elevation can be obtained as 


$$
\phi_{i}^{\varsigma}=\arccos \frac{\arg \left(\mu_{i}^{\varsigma}\right)}{\pi}, \quad i=1,2, \ldots, q,
$$

where $\mu_{i}^{\varsigma}$ is the $i^{\text {th }}$ eigenvalue of $\mathbf{W}_{2 \varsigma}^{1}\left(\mathbf{W}_{2 \varsigma-1}^{1}\right)^{+}$, and superscript $\varsigma$ of $\mu_{i}^{\varsigma}$ denotes the eigenvalue calculated by $\mathbf{W}_{2 \varsigma-1}^{1}$ and $\mathbf{W}_{2 \varsigma}^{1}$. The corresponding eigenvector of $\mu_{i}^{\varsigma}$ is $\xi_{i}^{\varsigma}$. Then, the eigenvalue $v_{i}^{\varsigma}$ of $\mathbf{W}_{2 \varsigma-1}^{2}\left(\mathbf{W}_{2 \varsigma-1}^{1}\right)^{+}$, which has the same position as $\mu_{i}^{\varsigma}$, can be expressed as

$$
v_{i}^{\varsigma}=\frac{1}{q} 1_{1 \times q} \cdot\left[\left(\frac{\mathbf{W}_{2 \varsigma-1}^{2}\left(\mathbf{W}_{2 \varsigma-1}^{1}\right)^{+} \boldsymbol{\xi}_{i}^{\varsigma}}{\xi_{i}^{\varsigma}}\right)\right],
$$

where $1_{1 \times q}$ is the $1 \times q$ dimensional vector with all the elements being 1 ; then, the nominal azimuth of the distribution source can be obtained as follows:

$$
\theta_{i}^{\varsigma}=\arccos \frac{\arg \left(\nu_{i}^{\varsigma}\right)}{\pi \sin \phi_{i}^{\varsigma}}, \quad i=1,2, \ldots, q .
$$

It can be concluded that the nominal elevation $\phi_{i}$ and the nominal azimuth $\theta_{i}$ are the mean values obtained from the $K / 2$ group of $\mathbf{W}_{2 \varsigma-1}^{1}, \mathbf{W}_{2 \varsigma^{-1}}^{2}$, and $\mathbf{W}_{2 \varsigma}^{1}$.

$$
\begin{gathered}
\phi_{i}=\frac{2}{K} \sum_{\zeta=1}^{K / 2} \phi_{i}^{\varsigma}, \\
\theta_{i}=\frac{2}{K} \sum_{\zeta=1}^{K / 2} \theta_{i}^{\varsigma} .
\end{gathered}
$$

\section{DOA Tracking Based on FAPI}

On the basis of obtaining the signal subspace, the DOA tracking of $2 \mathrm{D}$ CD signal sources can be realized by the above approach proposed. In this part, the signal subspace is updated in real time by FAPI algorithm under URA; then, DOA is solved by the estimation algorithm in the previous part. In this way, real-time tracking of $\mathrm{DOA}$ of $2 \mathrm{D} \mathrm{CD}$ sources can be realized.

For the tracking problem, the sample covariance matrix of snapshot data $\mathbf{X}(t)(t=1,2, \ldots, N)$ can be recursively updated in form of an exponential window as follows:

$$
\mathbf{R}(t)=\alpha \mathbf{R}(t-1)+\mathbf{X}(t) \mathbf{X}(t)^{H}
$$

where $a>0$ is the forgetting factor. Define $\mathbf{W}(t)$ is the signal subspace of the sample covariance matrix at time $t$. Then, the power iteration method can be written follows:

$$
\mathbf{W}(t)=\mathbf{R}(t) \mathbf{W}(t-1) .
$$

In order to ensure that the modulus of each vector in the signal subspace is 1 , multiply the right end of equation (36) by a coefficient matrix to obtain the following relationship:

$$
\mathbf{W}(t)=\mathbf{R}(t) \mathbf{W}(t-1)\left[\mathbf{W}(t-1){ }^{H} \mathbf{R}^{2}(t-1) \mathbf{W}(t-1)\right]^{-1 / 2} .
$$

Equation (37) can guarantee the orthogonality of $\mathbf{W}(t)$ in the iterative process, that is to say

$$
\mathbf{W}(t){ }^{H} \mathbf{W}(t)=\mathbf{I}_{q} .
$$

Authors of [16] have proved that expression (38) converges to the signal subspace of the covariance matrix.

Define compressed signal vector as

$$
\mathbf{V}(t)=\mathbf{W}(t-1)^{H} \mathbf{X}(t) .
$$

Then, equation (37) can be expressed as

$$
\mathbf{W}(t) \Psi(t)=\mathbf{R}_{W}(t),
$$

where

$$
\mathbf{R}_{\mathrm{W}}(t)=\mathbf{R}(t) \mathbf{W}(t-1) .
$$

$\mathbf{R}_{\mathrm{w}}(t)$ can be regarded as the cross covariance matrix of the received signal vector $\mathrm{X}(t)$ and the compressed signal vector $\mathbf{V}(t) . \Psi(t)$ is the square root matrix of $\mathbf{R}_{\mathrm{w}}(t)$, which have the following expression:

$$
\boldsymbol{\Psi}(t)=\left[\mathbf{R}_{W}(t)^{H} \mathbf{R}_{W}(t)\right]^{1 / 2} .
$$

The power iterative method can be composed of the data compression process (39) and orthogonalization process (40). Although the power iterative method is very effective, the calculation complexity of the orthogonal process is high, which is not conducive to real-time application. This classical iterative subspace estimation method often cannot guarantee the good convergence and robust performance of the results.

FAPI algorithm is proposed in [17], which are optimized and solved on the basis of the power iteration method. In order to track the signal subspace $\mathbf{W}(t)$, the detailed steps of FAPI algorithm are summarized in Table 1.

The proposed 2D DOA fast tracking algorithm steps are listed as follows.

(1) Initialize $\mathbf{W}(0)$ and $\mathbf{L}(0)$

(2) Update snapshot data $\mathbf{X}(t)$ and obtain the signal subspace $\mathbf{W}(t)$ at time $t$ by the FAPI algorithm described in Table 1

(3) Divide the subspace into $K / 2$ groups of $\mathbf{W}_{2 \varsigma-1}^{1}, \mathbf{W}_{2 \varsigma-1}^{2}$, and $\mathbf{W}_{2 \varsigma}^{1}$

(4) Calculate the nominal elevation from the eigenvalue obtained by eigendecomposition using equation (30) and calculate the azimuth angle using equations (31) and (32)

(5) Obtained the mean value by equations (33) and (34) as the estimated elevation and azimuth

It can be seen from table that the computational complexity required for each iteration of signal subspace updating is $\mathrm{O}(M K q)$, which is a very low value. After the signal subspace is obtained, the complexity of DOA estimation mainly lies in the eigendecomposition of $\mathrm{R}_{\mathrm{Z}}$, which is $O\left[(M K)^{3}\right]$, and calculate a pseudoinverse of $\mathbf{W}_{2 \zeta-1}^{1}$, which is $\mathrm{O}\left[(M K)^{3}\right]$. 
TABLE 1: Pseudocode of the FAPI method.

Input: $\{\mathbf{X}(t)\}_{t=1}^{N}$, which is $N$ snapshots of receive vectors of URA.

Output: $\mathrm{W}(t)$, which is the signal subspace of the covariance matrix of $\mathbf{X}(t)$.

Initialize: $\mathbf{W}(0)=\left[\mathbf{I}_{q \times q}, 0_{(M K-q) \times q}\right]^{T}, \mathbf{L}(0)=\mathbf{I}_{q \times q}$.

1. $\mathbf{V}(t)=\mathbf{W}(t-1)^{H} \mathbf{X}(t)$

2. $\mathbf{g}(t)=\mathbf{L}(t-1) \mathbf{V}(t)\left[\alpha+\mathbf{V}(t)^{H} \mathbf{L}(t-1)^{H} \mathbf{V}(t)\right]^{-1}$

3. $\gamma(t)^{2}=\mathbf{X}(t)^{H} \mathbf{X}(t)-\mathbf{V}(t)^{H} \mathbf{V}(t)$

4. $\beta(t)=1+\gamma(t)^{2}\|\mathbf{g}(t)\|^{2}$

5. $\tau(t)=\gamma(t)^{2}\left[\beta(t)+\beta(t)^{1 / 2}\right]^{-1}$

6. $\xi(t)=1-\tau(t)\|\mathbf{g}(t)\|^{2}$

7. $\mathbf{X}^{\prime}(t)=\mathbf{V}(t) \xi(t)+\mathbf{g}(t) \tau(t)$

8. $\varsigma(t)=\tau(t) \eta(t)^{-1}\left[\mathbf{L}(t-1) \mathbf{g}(t)-\mathbf{g}(t) \mathbf{X}^{\prime}(t)^{H} \mathbf{g}(t)\right]$

9. $\mathbf{L}(t)=\alpha^{-1}\left[\mathbf{L}(t-1)-\mathbf{g}(t) \mathbf{X}^{\prime}(t)^{H} \mathbf{L}(t-1)+\varsigma(t) \mathbf{g}(t)^{H}\right]$

10. $\mathbf{e}^{\prime}(t)=\mathbf{X}(t) \xi(t)-\mathbf{W}(t-1) \mathbf{g}(t) \tau(t)$

11. $\mathbf{W}(t)=\mathbf{W}(t-1)+\mathbf{e}^{\prime}(t) \mathbf{g}(t)^{H}$

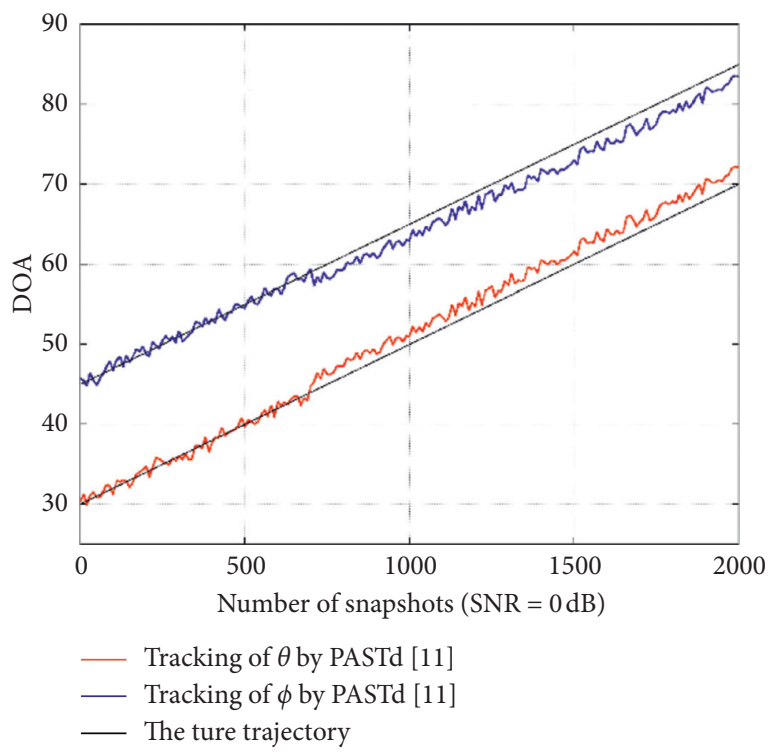

(a)

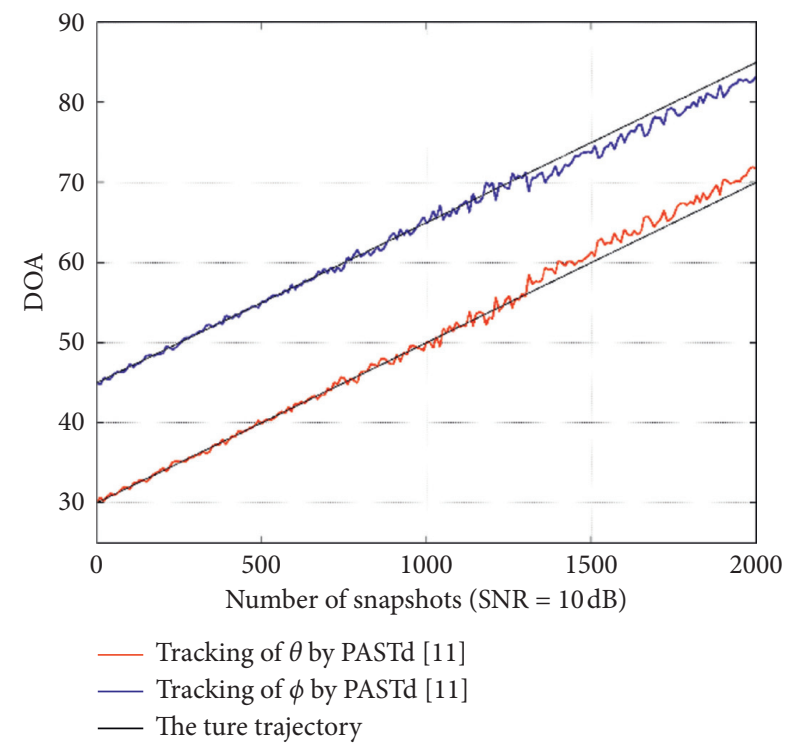

(b)

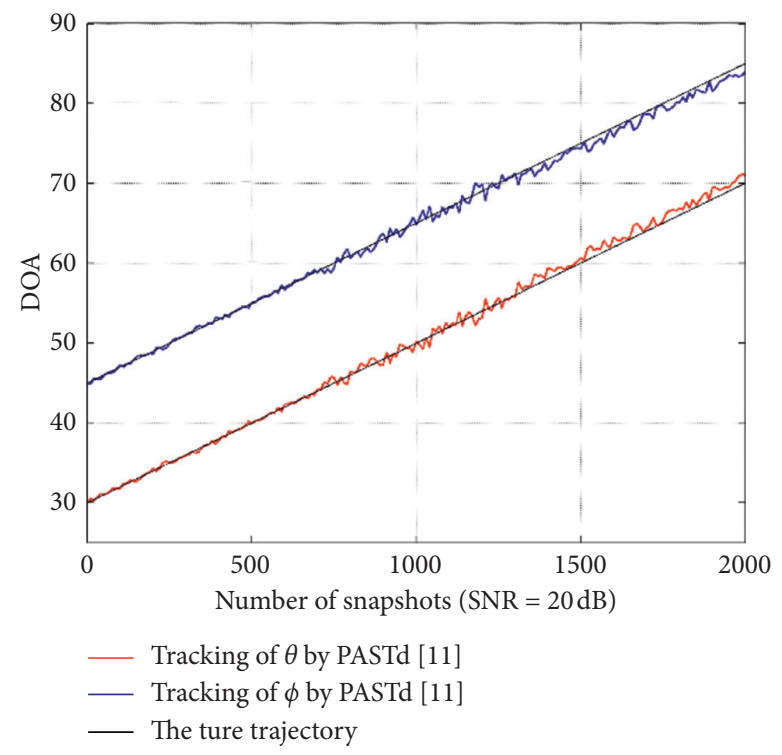

(c)

FIGURE 2: DOA tracking of a 2D CD source by PASTd for the point source. 


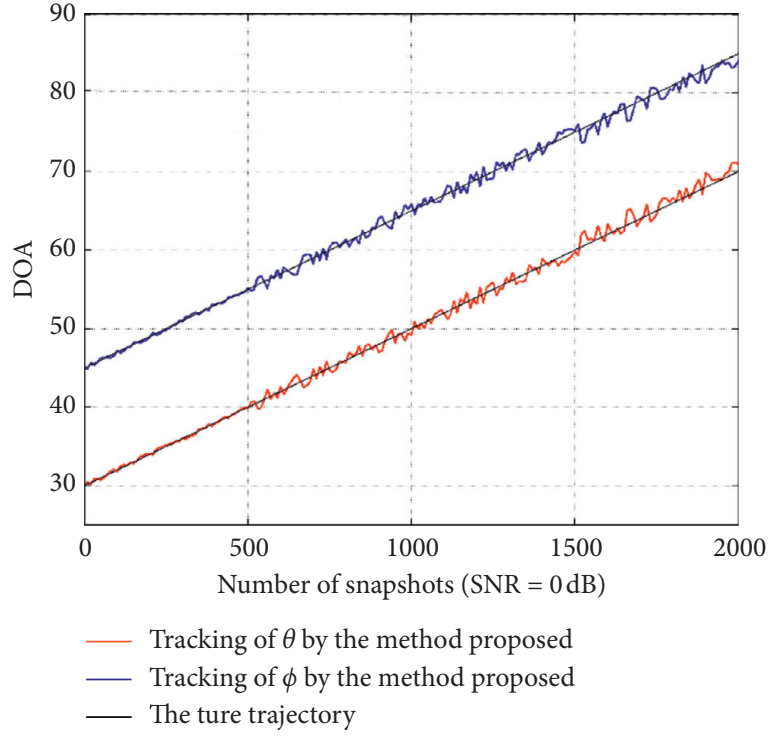

(a)

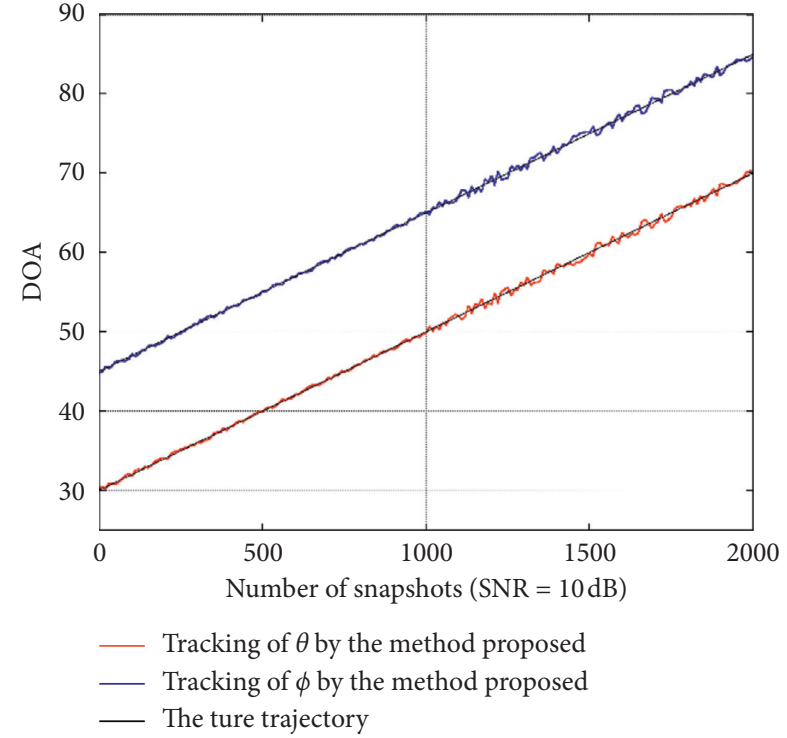

(b)

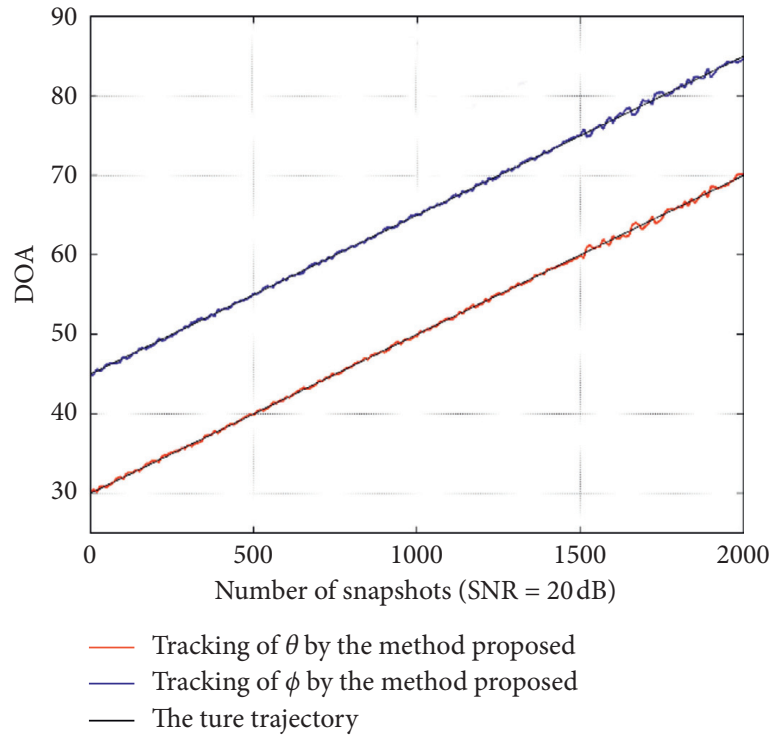

(c)

FIgURE 3: DOA tracking of a 2D CD source by the method proposed.

\section{Results and Discussion}

In this section, three experiments are conducted to investigate the tracking performance of the proposed algorithm. The array model shown in Figure 1 is used. The array geometric parameters are $M=K=4$, and $d=0.5 \lambda$. The forgetting factor $\alpha=0.95$. It is assumed that the angular spread parameters remain unchanged during the movement of distributed sources.

Experiment 1 investigates the influence of the signal-tonoise ratio (SNR) on DOA tracking of single CD source and effectiveness of the method proposed. The initial DOA parameters are $\left(30^{\circ}, 45^{\circ}\right)$ and transformed to $\left(70^{\circ}, 85^{\circ}\right)$ during 2000 snapshots uniformly. Figure 2 shows the tracking curves by PASTd [11] with $\mathrm{SNR}=0 \mathrm{~dB}, 10 \mathrm{~dB}$, and $20 \mathrm{~dB}$.
Figure 3 shows the tracking curves of the algorithm proposed when the angular spread is $2^{\circ}$. It can be seen that with the increase of SNR, the tracking effect of both algorithms is improved. The experimental results show that the proposed algorithm has a good tracking performance for the single $2 \mathrm{D}$ $\mathrm{CD}$ source. It is worth noting that even with the increase of SNR, both the estimated nominal elevation and nominal azimuth have a stable trend error with the passage of time in Figure 2, that is, the estimated nominal elevation is less than the true value and the estimated nominal azimuth is greater than the real value. Since PASTd [11] is for DOA tracking of the point source, when the target model is a distributed source, there is a large error due to model mismatch.

Experiment 2 examines the effect of angular spread on DOA tracking of the single CD source. The changing process 


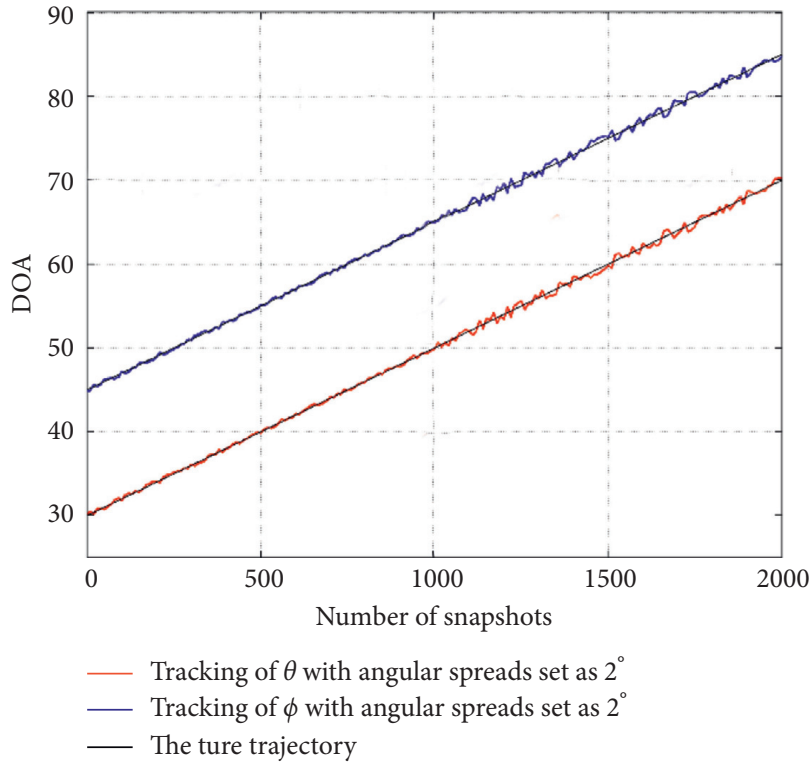

(a)

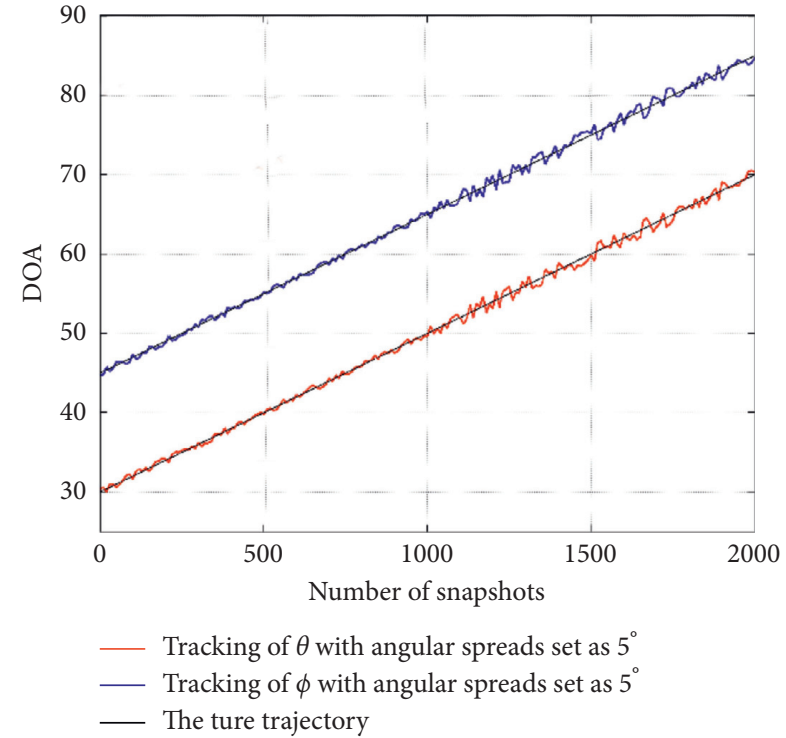

(b)

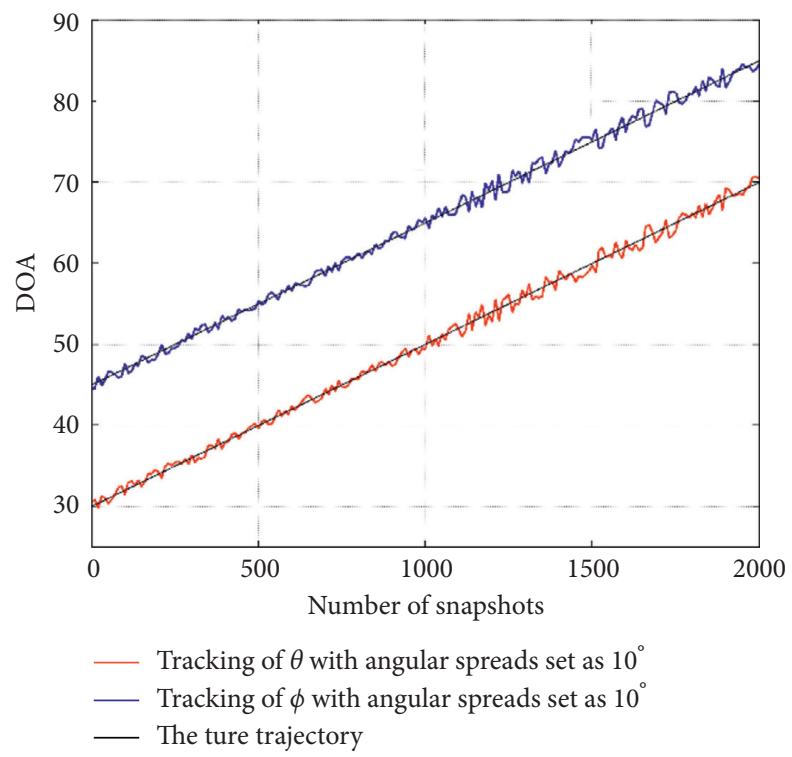

(c)

FIGURE 4: DOA tracking of a 2D CD source with different angular spreads.

of DOA is the same as experiment 1 . Figure 4 shows tracking curves with angular spreads set as $2^{\circ}, 5^{\circ}$, and $10^{\circ}$ when $\mathrm{SNR}=10 \mathrm{~dB}$. It can be seen that the tracking effect decreases with the increase of the angular spreads. The derivation of the rotating invariant relations in the DOA estimation stage is based on the assumption of the small angular spreads, so the increase of the angular spread will bring errors in the DOA estimation step. As a matter of fact, whether it is Gaussian or a uniform distributed source or any other distributed sources, when the angular spreads are $0^{\circ}$, the parameters describing the $\mathrm{CD}$ source only include DOA parameters containing azimuth and elevation, which means the distributed source can be considered as a point source. This experiment also shows that the method proposed in this paper is not only suitable for DOA tracking of the CD source but also suitable for DOA tracking of the point source. In the case of mismatch between the point source and CD source model, it has good robustness.

Experiment 3 investigates DOA tracking effectiveness for multiple sources. Assuming that three CD sources are incident on the array, all the angular spreads of the sources are $2^{\circ}$. The ASDF of the first CD source obeys Gaussian distribution, while the second and the third CD source obey the uniform distribution. The first source is transformed from $\left(30^{\circ}, 45^{\circ}\right)$ to $\left(70^{\circ}, 25^{\circ}\right)$ in 2000 snapshots. The second and third sources are transformed from $\left(40^{\circ}, 45^{\circ}\right)\left(50^{\circ}, 45^{\circ}\right)$ to $\left(80^{\circ}, 25^{\circ}\right)\left(90^{\circ}, 25^{\circ}\right)$ in 2000 snapshots, respectively. Figure 5 shows tracking curves of three sources based on the method proposed. From the derivation process in parts 3 and 4 and experimental results, we can see that the 


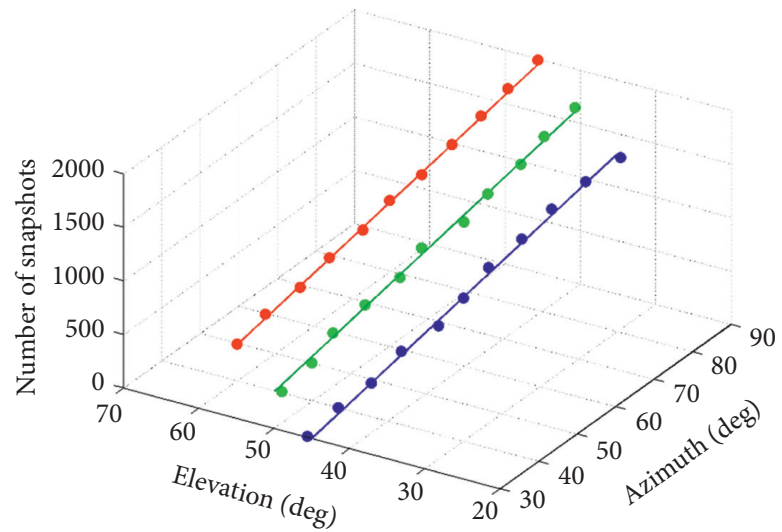

FIGURE 5: DOA tracking of multiple CD sources by the method proposed.
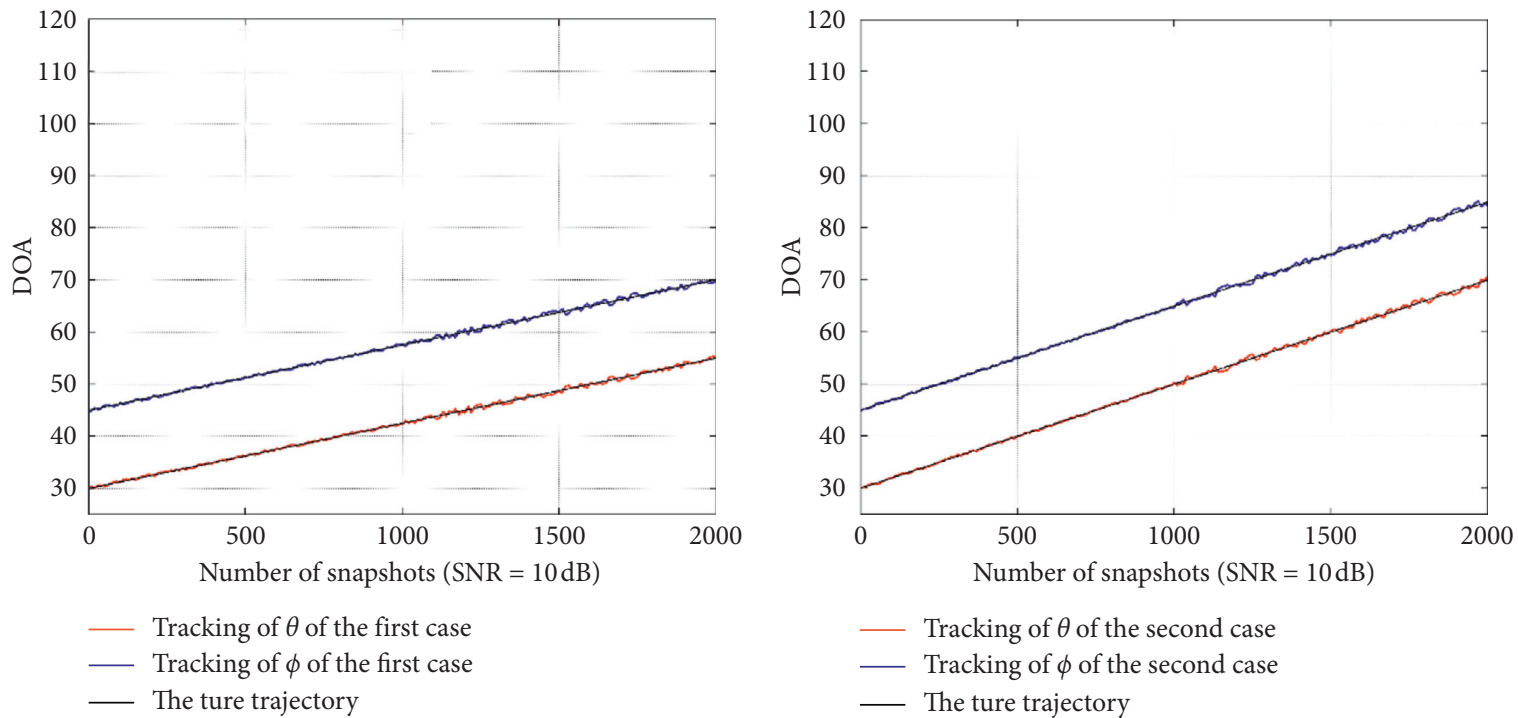

(b)
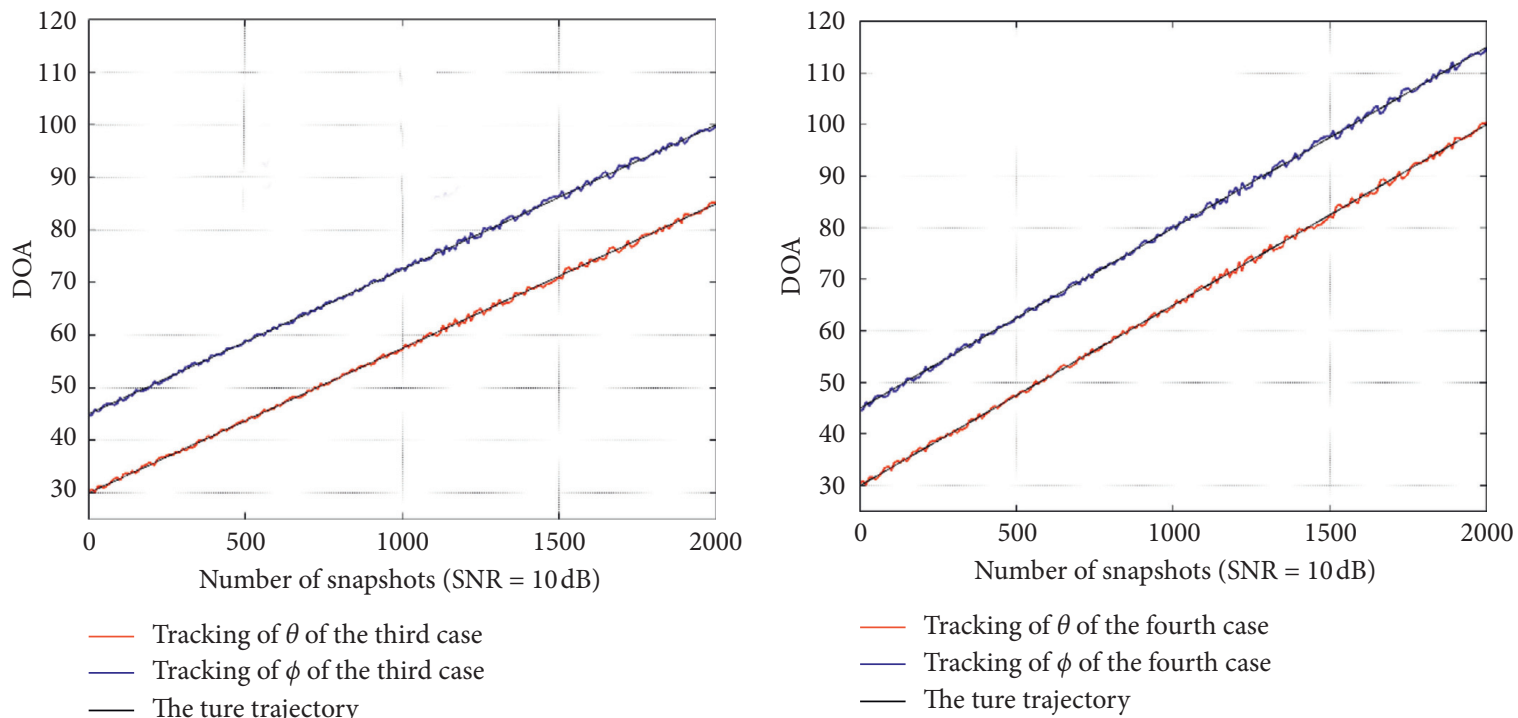

(c)

(d)

FIGURE 6: DOA tracking under different change rates. 
method proposed in this paper does not need to know the specific form of ASDF of sources during the DOA tracking process. The results show that the algorithm can effectively track the DOA of multiple distribution sources without ASDF information.

Experiment 4 investigates the tracking effect of the proposed algorithm under different change rates when the distributed source changes uniformly. All the CD sources are Gaussian type, the angular spreads are all $2^{\circ}$, and $\mathrm{SNR}=10 \mathrm{~dB}$. The transformation processes are in 2000 snapshots. Four kinds of transformation are investigated. The first case is that the source changes from $\left(30^{\circ}, 45^{\circ}\right)$ to $\left(55^{\circ}, 70^{\circ}\right)$; the second case changes from $\left(30^{\circ}, 45^{\circ}\right)$ to $\left(70^{\circ}\right.$, $\left.85^{\circ}\right)$; the third case changes from $\left(30^{\circ}, 45^{\circ}\right)$ to $\left(85^{\circ}, 100^{\circ}\right)$; and the fourth case changes from $\left(30^{\circ}, 45^{\circ}\right)$ to $\left(100^{\circ}, 115^{\circ}\right)$. It can be seen from Figure 6 that with the increase of the change rates, the tracking accuracy will slightly deteriorate; the FAPI algorithm is based on the updating of the sample covariance matrices in the form of an exponential window, which is the approximation of the covariance matrices of moving targets. But on the whole, the tracking effect is still satisfactory.

\section{Conclusions}

In this paper, a fast tracking algorithm based on subspace updating is proposed for moving 2D CD sources under URA. The signal subspace is updated by fast approximated power iteration algorithm; the nominal azimuth and nominal elevation are calculated by an ESPRIT-like algorithm through the estimation synthesis of multiple groups of parallel linear arrays within URA arrays. The algorithm proposed in this paper has low complexity and is suitable for real-time tracking. Experimental results show that the algorithm proposed in this paper has high tracking accuracy and robustness in case of mismatch between the point source and CD source model and can track multiple distribution sources without knowing the angular signal distribution functions.

\section{Appendix}

Define $\tilde{\theta}$ as the small deviations of $\theta_{i}$ and $\widetilde{\phi}$ as the small deviations of $\varphi_{i}$. Replace $\theta$ with $\left(\theta_{i}+\widetilde{\theta}\right)$ and $\varphi$ with $\left(\phi_{i}+\widetilde{\phi}\right)$; we can obtain the approximate representations of $\cos \theta \sin \varphi$ and $\cos \varphi$ utilizing the first term of the Taylor series expansions. Consider both sides of equation (8).

$$
\begin{aligned}
& \eta_{m k}\left(\theta_{i}, \phi_{i}\right)=\iint e^{j \pi(m-1) \cos \theta \sin \phi} e^{j \pi(k-1) \cos \phi} g_{i}\left(\theta, \phi ; \mathbf{u}_{i}\right) d \theta d \phi, \\
& =\iint e^{j \pi(m-1)\left(\cos \theta_{i} \sin \phi_{i}+\cos \theta_{i} \cos \tilde{\phi}_{i} \tilde{\phi}-\sin \theta_{i} \sin \phi_{i} \tilde{\theta}\right)} e^{j \pi(k-1)\left(\cos \phi_{i}-\sin \phi_{i} \widetilde{\phi}\right)} g_{i}\left(\theta, \phi ; \mathbf{u}_{i}\right) d \widetilde{\theta}, \\
& =e^{j \pi(m-1) \cos \theta_{i} \sin \phi_{i}} e^{j \pi(k-1) \cos \phi_{i}} \iint e^{j \pi(m-1)\left(\cos \theta_{i} \cos \phi_{i} \tilde{\phi}-\sin \theta_{i} \sin \phi_{i} \tilde{\theta}\right)} e^{j \pi(k-1) \sin \phi_{i} \tilde{\phi}} g_{i}\left(\theta, \phi ; \mathbf{u}_{i}\right) d \tilde{\theta} \text {. } \\
& \eta_{(m-1) k}\left(\theta_{i}, \phi_{i}\right)=\iint e^{j \pi(m-2)(\cos \theta \sin \phi)} e^{j(k-1) \cos \phi} g_{i}\left(\theta, \phi ; \mathbf{u}_{i}\right) d \theta d \phi, \\
& =\iint e^{j \pi(m-2)\left(\cos \theta_{i} \sin \phi_{i}+\cos \theta_{i} \cos \phi_{i} \widetilde{\phi}-\sin \theta_{i} \sin \phi_{i} \tilde{\theta}\right)} e^{j \pi(k-1)\left(\cos \phi_{i}-\sin \phi_{i} \widetilde{\phi}\right)} g_{i}\left(\theta, \phi ; \mathbf{u}_{i}\right) d \widetilde{\theta}, \\
& =e^{j \pi(m-2) \cos \theta_{i} \sin \phi_{i}} e^{j \pi(k-1) \cos \phi_{i}} \iint e^{-j \pi\left(\cos \theta_{i} \cos \phi_{i} \widetilde{\phi}-\sin \theta_{i} \sin \phi_{i} \tilde{\theta}\right)} e^{j \pi(m-1)\left(\cos \theta_{i} \cos \phi_{i} \tilde{\phi}-\sin \theta_{i} \sin \phi_{i} \tilde{\theta}\right)} e^{j \pi(k-1) \sin \phi_{i} \tilde{\phi}} \\
& \text { - } g_{i}\left(\theta, \phi ; \mathbf{u}_{i}\right) d \tilde{\theta} .
\end{aligned}
$$

$$
\eta_{m k}\left(\theta_{i}, \phi_{i}\right) \approx e^{j \pi \cos \theta_{i} \sin \phi_{i}} \eta_{(m-1) k}\left(\theta_{i}, \phi_{i}\right) .
$$

As $e^{-j \pi\left(\cos \theta_{i} \cos \phi_{i} \tilde{\phi}-\sin \theta_{i} \sin \phi_{i} \tilde{\theta}\right)} \approx 1$, the following equation can be obtained.

Consider the right side of equation (9).

$$
\begin{aligned}
\eta_{m(k-1)}\left(\theta_{i}, \phi_{i}\right) & =\iint e^{j(m-1)(\cos \theta \sin \phi)} e^{j(k-2) \cos \phi} g_{i}\left(\theta, \phi ; \mathbf{u}_{i}\right) d \theta d \phi \\
& =\iint e^{j \pi(m-2)\left(\cos \theta_{i} \sin \phi_{i}+\cos \theta_{i} \cos \phi_{i} \tilde{\phi}-\sin \theta_{i} \sin \phi_{i} \widetilde{\theta}\right)} e^{j \pi(k-2)\left(\cos \phi_{i}-\sin \phi_{i} \widetilde{\phi}\right)} g_{i}\left(\theta, \phi ; \mathbf{u}_{i}\right) d \widetilde{\theta}, \\
& =e^{j \pi(m-1) \cos \theta_{i} \sin \phi_{i}} e^{j \pi(k-2) \cos \phi_{i}} \iint e^{-j \pi \sin \phi_{i} \widetilde{\phi}} e^{j \pi(m-1)\left(\cos \theta_{i} \cos \phi_{i} \tilde{\phi}-\sin \theta_{i} \sin \phi_{i} \tilde{\theta}\right)} e^{j \pi(k-1) \sin \phi_{i} \tilde{\phi}} g_{i}\left(\theta, \phi ; \mathbf{u}_{i}\right) d \widetilde{\theta}
\end{aligned}
$$

Noticing $e^{-j \pi \sin \phi_{i} \tilde{\phi}} \approx 1$, we obtain the relation between $\eta_{m k}\left(\theta_{i}, \phi_{i}\right)$ and $\eta_{m(k-1)}\left(\theta_{i}, \phi_{i}\right)$.

$$
\eta_{m k}\left(\theta_{i}, \phi_{i}\right) \approx e^{j \pi \cos \phi_{i}} \eta_{m(k-1)}\left(\theta_{i}, \phi_{i}\right)
$$




$$
\eta_{m k}\left(\theta_{i}, \phi_{i}\right) \approx e^{j \pi \cos \theta_{i} \sin \phi_{i}} e^{j \pi \cos \phi_{i}} \eta_{(m-1)(k-1)}\left(\theta_{i}, \phi_{i}\right) . \quad \text { A.6 }
$$

\section{Data Availability}

The data used to support the findings of this study are available from the corresponding author upon request.

\section{Conflicts of Interest}

The authors declare that they have no conflicts of interest.

\section{Acknowledgments}

The authors would like to thank the editorial board and reviewers for the improvement of this paper. This research was funded by the National Natural Science Foundation of China (grant nos. 51776222 and 61903378) and Field Foundation of China (grant no. 61400020109).

\section{References}

[1] H. Chen, C. Hou, W.-P. Zhu et al., "ESPRIT-like two-dimensional direction finding for mixed circular and strictly noncircular sources based on joint diagonalization," Signal Processing, vol. 141, pp. 48-56, 2017.

[2] X. Wang, L. Wan, M. Huang, C. Shen, Z. Han, and T. Zhu, "Low-complexity channel estimation for circular and noncircular signals in virtual MIMO vehicle communication systems," IEEE Transactions on Vehicular Technology, vol. 69, no. 4, pp. 3916-3928, 2020.

[3] Z. Wang, X. F. Zhang, Y. F. Wang et al., "Extended coprime array configuration generating large-scale antenna co-array in massive MIMO system," IEEE Transactions on Vehicular Technology, vol. 99, pp. 7841-7852, 2019.

[4] Z. G. Shi, C. W. Zhou, Y. J. Gu et al., "Source estimation using coprime array: a sparse reconstruction perspective," IEEE Sensors Journal, vol. 17, pp. 755-765, 2016.

[5] F. Wen, J. Shi, and Z. Zhang, "Joint 2D-DOD, 2D-DOA, and Polarization angles estimation for bistatic EMVS-MIMO radar via PARAFAC analysis," IEEE Transactions on Vehicular Technology, vol. 69, no. 2, pp. 1626-1638, 2020.

[6] X. Zhang, L. Xu, L. Xu, and D. Xu, "Direction of departure (DOD) and direction of arrival (DOA) estimation in MIMO radar with reduced-dimension MUSIC," IEEE Communications Letters, vol. 14, no. 12, pp. 1161-1163, 2010.

[7] F. Wen and J. Shi, "Fast direction finding for bistatic EMVSMIMO radar without pairing," Signal Processing, vol. 173, pp. 107512-107518, 2020.

[8] F. Wen, Z. Zhang, K. Wang, G. Sheng, and G. Zhang, "Angle estimation and mutual coupling self-calibration for ULAbased bistatic MIMO radar," Signal Processing, vol. 144, pp. 61-67, 2018.

[9] A. J. Sanchez and S. Marcos, "An efficient PASTd-algorithm implementation for multiple direction of arrival tracking," IEEE Signal Process, vol. 47, pp. 2321-2324, 2002.

[10] C. R. Rao, C. R. Sastry, and B. Zhou, "Tracking the direction of arrival of multiple moving targets," IEEE Transactions on Signal Processing, vol. 42, no. 5, pp. 1133-1144, 1994.

[11] C.-S. Ryu, S.-H. Lee, and K.-K. Lee, "Multiple target angle tracking algorithm using angular innovations extracted from signal subspace," Electronics Letters, vol. 35, no. 18, pp. $1520-1522,1999$.
[12] B. Yang, "Projection approximation subspace tracking," IEEE Signal Process, vol. 43, pp. 95-107, 1995.

[13] B. Yang, "Convergence analysis of the subspace tracking algorithms PAST and PASTd," in Proceedings of IEEE International Conference on Acoustics, Speech, \& Signal Processing, Icassp-96 Conference, Atlanta, GA, USA, May 1996.

[14] A. J. Sanchez and S. Marcos, "An efficient PASTd-algorithm implementation for multiple direction of arrival tracking," IEEE Signal Process, vol. 47, pp. 2321-2324, 1999.

[15] M. K. Abed, A. Chkeif, and Y. Hua, "Fast orthonormal PAST algorithm," IEEE Signal Processing Letters, vol. 7, pp. 60-62, 2000.

[16] Y. Hua, Y. Xiang, T. Chen, K. Abed-Meraim, and Y. Miao, "A new look at the power method for fast subspace tracking," Digital Signal Processing, vol. 9, no. 4, pp. 297-314, 1999.

[17] R. Badeau, B. David, and G. Richard, "Fast approximated power iteration subspace tracking," IEEE Transactions on Signal Processing, vol. 53, no. 8, pp. 2931-2941, 2005.

[18] S. Valaee, B. Champagne, and P. Kabal, "Parametric localization of distributed sources," IEEE Transactions on Signal Processing, vol. 43, no. 9, pp. 2144-2153, 1995.

[19] Z. Zheng, W.-Q. Wang, H. Meng, H. C. So, and H. Zhang, "Efficient beamspace-based algorithm for two-dimensional DOA estimation of incoherently distributed sources in massive MIMO systems," IEEE Transactions on Vehicular Technology, vol. 67, no. 12, pp. 11776-11789, 2018.

[20] Z. Dai, W. Cui, B. Ba, D. Wang, and Y. Sun, "Two-dimensional DOA estimation for coherently distributed sources with symmetric properties in crossed arrays," Sensors, vol. 17, p. 1300, 2017.

[21] C. Wen, G. Shi, and X. Xie, "Estimation of directions of arrival of multiple distributed sources for nested array," Signal Processing, vol. 130, pp. 315-322, 2017.

[22] X. Yang, Z. Zheng, C. C. Ko, and L. Zhong, "Low-complexity 2D parameter estimation of coherently distributed noncircular signals using modified propagator," Multidimensional Systems and Signal Processing, vol. 28, no. 2, pp. 407-426, 2017.

[23] L. Wan, G. Han, J. Jiang, C. Zhu, and L. Shu, "A DOA estimation approach for transmission performance guarantee in D2D Communication," Mobile Networks and Applications, vol. 22, no. 6, pp. 998-1009, 2017.

[24] Z. Zheng, G. Li, and Y. Teng, "2D DOA estimator for multiple coherently distributed sources using modified propagator," Circuits, Systems, and Signal Processing, vol. 31, no. 1, pp. 255-270, 2012.

[25] Z. Zheng, G. Li, and Y. Teng, "Low-complexity 2D DOA estimator for multiple coherently distributed sources," COMPEL-The International Journal for Computation and Mathematics in Electrical and Electronic Engineering, vol. 31, no. 2, pp. 443-459, 2012.

[26] A. Zoubir and Y. Wang, "Performance analysis of the generalized beamforming estimators in the case of coherently distributed sources," Signal Processing, vol. 88, no. 2, pp. 428-435, 2008.

[27] K. Han and A. Nehorai, "Nested array processing for distributed sources," IEEE Signal Processing Letters, vol. 21, pp. 1111-1114, 2014.

[28] X. Yang, G. Li, Z. Zheng, and L. Zhong, "2D DOA estimation of coherently distributed noncircular sources," Wireless Personal Communications, vol. 78, no. 2, pp. 1095-1102, 2014.

[29] W. Xiong, P. José, and M. Sylvie, "Performance analysis of distributed source parameter estimator (DSPE) in the 
presence of modeling errors due to the spatial distributions of sources," Signal Process, vol. 143, pp. 146-151, 2018.

[30] W. Xiong, J. Picheral, and S. Marcos, "Robustness of the coherently distributed MUSIC algorithm to the imperfect knowledge of the spatial distribution of the sources," Signal, Image and Video Processing, vol. 11, no. 4, pp. 721-728, 2017.

[31] S. B. Hassen and A. Samet, "An efficient central DOA tracking algorithm for multiple incoherently distributed sources," EURASIP Journal on Advances in Signal Processing, vol. 2015, no. 1, pp. 1-19, 2015.

[32] Y. Han, J. Wang, and Q. Zhao, "An efficient DOA tracking algorithm for coherently distributed source," in Proceedings of the 2011 11th International Symposium on Communications \& Information Technologies, Hangzhou, China, October 2011.

[33] X. S. Guo, Q. Wan, and W. L. Yang, "Fast DOA tracking of coherently distributed sources based on subspace updating," in Proceedings of the 2006 CIE International Conference on Radar, Shanghai, China, October 2006. 